\title{
The role of increased pulmonary blood flow in pulmonary arterial hypertension
}

\author{
M.E. van Albada*\#, R.G. Schoemaker*, M.S. Kemna", A.H. Cromme-Dijkhuis", \\ R. van Veghel $^{+}$and R.M.F. Berger*,\#
}

ABSTRACT: Chronic increased pulmonary blood flow is considered a pre-requisite for the induction of advanced vascular lesions in pulmonary arterial hypertension in congenital heart defects. The aim of the present study was to characterise the effects of increased pulmonary flow induced by an aortocaval shunt in the monocrotaline rat model for pulmonary hypertension in terms of survival, haemodynamics, pathology and histology.

Male Wistar rats were injected with monocrotaline followed by the creation of an abdominal aortocaval shunt. Animals were sacrificed when displaying symptoms of weight loss or dyspnoea, 4-5 weeks after the creation of the shunt.

Echocardiography identified increased ventricular dimensions in shunted rats and right ventricular hypertrophy in monocrotaline-treated rats. At similar pulmonary artery pressures, shunted monocrotaline rats displayed higher morbidity and mortality, increased pulmonary-tosystemic artery pressure ratios and increased right ventricular hypertrophy compared with nonshunted monocrotaline rats. Histological assessment demonstrated increased number and diameter of pre-acinar pulmonary arteries. Intra-acinar vessel remodelling and occlusion occurred to a similar extent in shunted and nonshunted monocrotaline rats.

In conclusion, increased pulmonary blood flow in monocrotaline-induced pulmonary hypertension is associated with increased morbidity, mortality, and unfavourable haemodynamic and cardiac effects. These effects could be attributed to more pronounced right heart failure rather than to altered intra-acinar pulmonary vessel remodelling.

KEYWORDS: Animal model, congenital heart disease, plexogenic arteriopathy, pulmonary arterial hypertension, pulmonary vascular histopathology, right heart failure

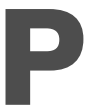

atients with congenital heart disease associated with systemic-to-pulmonary shunts, increasing both pulmonary blood flow and pressure, develop characteristic pulmonary vascular lesions as concentric-laminar intimal fibrosis and plexiform lesions. These vascular lesions are characterised by vascular smooth muscle and endothelial cell proliferation. In contrast, in patients with congenital heart defects and isolated increased pulmonary artery pressure $(P A P)$, these lesions almost never develop [1, 2]. The current authors hypothesise that, in congenital heart defects, increased pulmonary blood flow, in addition to increased pressure, is a pre-requisite for the development of the hallmark lesions of advanced pulmonary arterial hypertension.

Animal models with an isolated increase in PAP, e.g. induced by the toxic alkaloid monocrotaline, show pulmonary vascular remodelling, but fail to display the more advanced lesions as described previously [3-5].
The role of increased pulmonary blood flow has been explored in animal models [6]. In rat models with isolated increased flow $[7,8]$, a moderate increase in pulmonary pressure and medial hypertrophy has been observed, but only after a prolonged period of exposure.

When the additional effect of flow was explored in existing rat models for pulmonary arterial hypertension, cellular occlusion of small vessels, referred to as neo-intimal formation, was only observed in animals with increased pulmonary blood flow [9-11]. In contrast, one recent report suggests a favourable effect of increased blood flow. In this study, rats with an abdominal aortocaval shunt in addition to monocrotaline, showed a reduction in neo-intimal formation [10].

In the present study, the role of increased pulmonary flow induced by an abdominal aortocaval shunt in the monocrotaline rat model is explored in further detail via haemodynamical,
AFFILIATIONS

*Dept of Experimental Cardiology, University Medical Centre Groningen, and

\# Dept of Pediatrics, Division of Pediatric Cardiology, Beatrix Children's Hospital, University Medical Centre Groningen, Groningen, and "Dept of Pediatrics, Division of Pediatric Cardiology, and ${ }^{+}$Dept of Pharmacology, Erasmus Medical Center, Rotterdam, The Netherlands.

CORRESPONDENCE

M.E. van Albada

Dept of Experimental Cardiology University Medical Centre Groningen University of Groningen Antonius Deusinglaan 1 9713 AV Groningen The Netherlands

Fax: 31503632812

E-mail:

m.e.van.albada@med.umcg.nl

Received:

February 102005

Accepted after revision:

June 022005 
echocardiographical and histopathological characterisation, with special emphasis on neo-intimal formation.

\section{METHODS \\ Experimental protocol}

In total, 39 male Wistar rats (250-350 g; fed ad libitum) were housed under diurnal lighting conditions. Animal care and experiments were conducted according to the Dutch Animal Experimental Act. The Erasmus University Animal Care and Use Committee (Rotterdam, The Netherlands) approved the experimental protocol.

Rats were randomly assigned to one of four experimental groups: 1) control (CON: $n=11)$; 2) increased pulmonary flow by means of an abdominal aortocaval shunt (AV: $n=8) ; 3$ ) increased PAP, induced by monocrotaline (MC: $\mathrm{n}=8)$; and 4 ) the combination of both interventions (MC+AV: $n=12$ ). Monocrotaline (Sigma Chemical Co, St. Louis, MO, USA; $60 \mathrm{mg} \cdot \mathrm{kg}^{-1}$ subcutaneously) or vehicle were injected. Sham or AV-shunt surgery was performed 7 days later as described by GARCIA and DIEBOLD [12] under pentobarbital anaesthesia (60 mg $\mathrm{kg}^{-1}$ intraperitoneally). Shunt patency was verified visually as swelling, colour change and pulsation of the caval vein.

Animals were weighed, watched for dyspnoea and sacrificed when a $15 \%$ weight loss or debilitating dyspnoea occurred. Matched rats from the other groups were sacrificed simultaneously.

\section{Echocardiography}

In four rats from each group, echocardiographical studies were performed under pentobarbital anaesthesia before and 4 weeks after the administration of monocrotaline using a $12 \mathrm{MHz}$ phased array transducer (Sonos 5500; Hewlett-Packard Inc, Andover, MA, USA). Ventricular dimensions and flow profiles over the aortic, pulmonary and tricuspid valve were measured in standard views [13]. Cardiac output $\left(\mathrm{mL} \cdot \mathrm{min}^{-1}\right)$ was calculated using the following equation:

$$
\begin{aligned}
\text { cardiac output }= & (\text { aortic valve area }) \times(\text { aortic velocity } \\
& \text { time integral }) \times \text { heart rate }
\end{aligned}
$$

\section{Haemodynamics}

At sacrifice, animals were anesthetised and ventilated with room air. PAPs were measured according to RABINOVITCH et al. [14]. If these pressures could not be obtained, right ventricular systolic pressure was recorded as being equal to systolic PAP. Systemic arterial pressures were measured via the left carotid artery.

\section{Pathology}

After haemodynamic measurements, the thorax was opened and the presence of pleural fluid was noted. Atria, ventricles and lungs were weighed separately. The left lung was fixed in $3.6 \%$ formalin. Sections were stained with haematoxylin-eosin and resorcin-fuchsin elastic stain [15] for morphometric analysis of vascular dimensions using routine staining procedures. Additional sections were stained with antibodies against endothelial nitric oxide synthase (eNOS; dilution 1:500; BD Transduction Laboratories, Lexington, KY, USA) and $\alpha$-smooth muscle actin (Dako, Glostrup, Denmark). Paraffin sections were dewaxed and incubated with monoclonal antibodies for $1 \mathrm{~h}$. Rabbit anti-mouse immunoglobulin $\mathrm{G}$ coupled to peroxidase was used as a secondary antibody (dilution 1:100). The sections were stained with diaminobenzidine for $10 \mathrm{~min}$ and counterstained with haematoxylin.

\section{Histology}

In lung sections, all arteries $>50 \mu \mathrm{m}$ (pre-acinar) and 40 randomly chosen vessels $<50 \mu \mathrm{m}$ (intra-acinar) were assessed at $200 \times$ and $400 \times$ magnification using an image analysis system (CZ KS400; Imaging Associates, Bicester, UK). Three different vascular areas were defined: outer vessel area, inner vessel area and luminal area. The outer vessel area was defined as the area within the lamina elastica externa. The area within the lamina elastica interna was defined as the inner vessel area. The wall area was calculated by subtracting the luminal area from the outer vessel area. The medial area was defined as the outer vessel area minus the inner vessel area. The intimal area was calculated by subtracting the luminal area from the inner vessel area and expressed as a percentage of luminal area. Areas were transformed into diameters using the formula:

$$
\text { diameter }=2 \times \sqrt{ }(\text { area } / n)
$$

In the pre-acinar pulmonary arteries, the ratio between wall thickness and luminal diameter was calculated. Vessels $<50$ $\mu \mathrm{m}$ usually do not have a clearly discernible internal elastic lamina. Therefore, a vascular occlusion score was calculated in these vessels as opposed to the calculation of a medial wall to lumen ratio in the larger pulmonary arteries. Occlusion was calculated in the intra-acinar pulmonary vessels according to the following formula:

$$
\text { (outer vessel area-luminal area)/(outer vessel area) }
$$

Pulmonary arteries were excluded from measurement if they had a longest/shortest diameter of $>2$, an incomplete circular shape or a collapse of more than one quarter of the vessel wall. Muscularisation of 40 small pulmonary vessels was assessed according to VAN SUYLEN et al. [3].

\section{Statistical analysis}

Data are expressed as mean \pm SEM. Differences between groups were determined using one-way ANOVA with Bonferroni post hoc testing, using nonparametric tests when required (MannWhitney U-test and Kruskall-Wallis). Statistical significance was determined as $\mathrm{p}<0.05$.

\section{RESULTS}

Three rats had to be excluded from further analysis due to unsuccessful shunt surgery, two from the AV group and one from the $\mathrm{MC}+\mathrm{AV}$ group.

Reduced growth, lethargy and dyspnoea were observed in both the MC and MC+AV groups in the 5th and 6th week after monocrotaline administration. This led to the sacrifice of all rats in this period within a window of 1 week. Symptoms appeared earliest and the most severe in $\mathrm{MC}+\mathrm{AV}$ rats, leading to preliminary death in five out of 11 of these rats (table 1). 


\begin{tabular}{|c|c|c|c|}
\hline TABLE & $\begin{array}{c}\text { Animals with } \\
\text { excessive thorax } \\
\text { fluid }>12 \mathrm{~mL}\end{array}$ & $\begin{array}{c}\text { Animals that } \\
\text { determined } \\
\text { moment of sacrifice }\end{array}$ & $\begin{array}{l}\text { Body weight } \\
\text { at sacrifice }\end{array}$ \\
\hline CON & 0/11 (0) & $0 / 6(0)$ & $409 \pm 7$ \\
\hline AV & 0/6 (0) & 0/6 (0) & $429 \pm 24$ \\
\hline MC & 3/8 (37.5) & $1 / 6(16)$ & $360 \pm 6^{*}$ \\
\hline $\mathrm{MC}+\mathrm{AV}$ & $10 / 11(91)$ & $5 / 6(83)$ & $339 \pm 9^{*}$ \\
\hline \multicolumn{4}{|c|}{$\begin{array}{l}\text { Data are presented as mean } \pm \text { SEM or } n / n(\%) \text {. CON: healthy control animals; } \\
\text { AV: animals with increased pulmonary blood flow due to an aortocaval shunt; } \\
\mathrm{MC} \text { : animals with increased pulmonary arterial pressure due to monocrotaline } \\
\text { injections; } \mathrm{MC}+\mathrm{AV} \text { : animals with both increased pulmonary arterial pressure } \\
\text { and increased pulmonary blood flow, receiving both monocrotaline and an } \\
\text { aortocaval shunt. *: } p<0.05 \text { versus control animals. For the number of animals } \\
\text { that determined the moment of sacrifice, only the first six animals from each } \\
\text { group are shown, since the higher numbers did not have a matched control in } \\
\text { all other groups. }\end{array}$} \\
\hline
\end{tabular}

\section{Echocardiography}

Increased pulmonary flow due to the AV shunt was confirmed by an increased calculated cardiac output at 4 weeks compared with non-AV rats $(259 \pm 28$ versus $\left.150 \pm 12 \mathrm{~mL} \cdot \mathrm{min}^{-1} ; \quad \mathrm{p}=0.002\right)$. Furthermore, an increased diastolic left ventricular internal diameter $(8.52 \pm 0.22$ versus $6.73 \pm 0.34 \mathrm{~mm} ; \mathrm{p}=0.001)$, an increased diastolic right ventricular internal diameter $(5.78 \pm 0.20$ versus $4.15 \pm 0.33 \mathrm{~mm}$; $\mathrm{p}=0.001)$ and a significantly higher left ventricular mass $(1.17 \pm 0.06$ versus $0.95 \pm 0.03 \mathrm{~g} ; \mathrm{p}=0.004)$ were noted in the animals with a shunt compared with animals without a shunt.

Right ventricular wall thickness, an indication for increased right ventricular afterload, was increased in animals treated with monocrotaline compared with those without monocrotaline administration ( $1.06 \pm 0.11$ versus $0.72 \pm 0.03 \mathrm{~mm}$; $\mathrm{p}=0.02$ ). Three out of the four MC+AV rats developed a significant tricuspid regurgitant jet, which was not present at baseline, compared with none of the animals from the other groups.

\section{Haemodynamics}

Haemodynamic data are shown in figure 1 . Systolic $P$ AP (sPAP) was increased in the $\mathrm{MC}$ and $\mathrm{MC}+\mathrm{AV}$ groups, whereas systolic systemic arterial pressure ( $\mathrm{S} S \mathrm{AP}$ ) was only decreased in the $\mathrm{MC}+\mathrm{AV}$ group. Consequently, the $\mathrm{sPAP} / \mathrm{sSAP}$ ratio was increased in the MC+AV group compared with the other animal groups (fig. 1).

\section{Pathology}

Ventricular and atrial weights are shown in table 2. Heart weight to body weight ratios increased in all experimental groups with a significantly additive effect in the combined $\mathrm{MC}+\mathrm{AV}$ group.

Right ventricular to left ventricular plus septal weight ratio increased in the MC group with an additive effect of the shunt in the $\mathrm{MC}+\mathrm{AV}$ group (fig. 2).

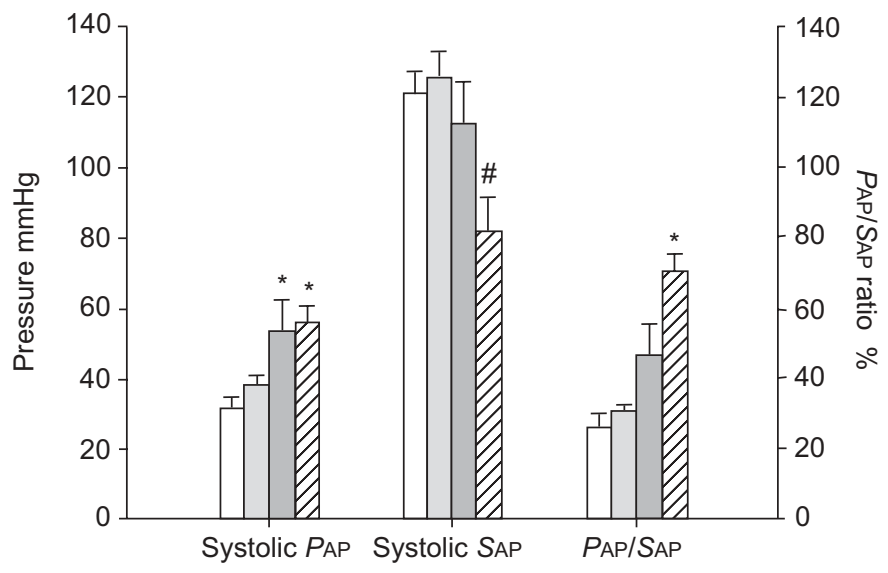

FIGURE 1. A bar chart demonstrating pulmonary arterial (PAP) and systemic arterial pressures (SAP) and PAP/SAP ratios in healthy control animals (CON $n=9$; $\square$ ), animals with increased pulmonary blood flow due to an aortocaval shunt (AV $\mathrm{n}=6 ; \quad-1)$, animals with increased pulmonary pressure due to monocrotaline injections ( $M C \mathrm{n}=5 ; \quad \square)$, and animals with both increased pulmonary artery pressure and increased pulmonary blood flow, receiving monocrotaline and an aortocaval shunt $(\mathrm{MC}+\mathrm{AV} \mathrm{n}=6 ; \mathbb{2})$. At sacrifice, systolic right ventricular and/or systolic PAP was obtained in a total of 26 rats. Sudden premature deaths in the MC group (one out of eight rats) and MC+AV group (five out of 11 rats) precluded invasive haemodynamic measurements. ${ }^{*}: p<0.05$ versus $\mathrm{CON}$; $\#$ : $p<0.05$ versus CON and AV.

\begin{tabular}{lcccc} 
TABLE 2 & \multicolumn{5}{c}{ Heart weights in grams } \\
& CON & AV & MC & MC+AV \\
\hline RV & $0.234 \pm 0.009$ & $0.368 \pm 0.017^{*}$ & $0.394 \pm 0.040^{*}$ & $0.523 \pm 0.023^{\#}$ \\
IVS & $0.305 \pm 0.012$ & $0.393 \pm 0.033^{\#}$ & $0.286 \pm 0.015$ & $0.309 \pm 0.015$ \\
LV & $0.526 \pm 0.022$ & $0.735 \pm 0.036^{\#}$ & $0.459 \pm 0.017$ & $0.516 \pm 0.034$ \\
RA & $0.029 \pm 0.003$ & $0.077 \pm 0.006^{*}$ & $0.053 \pm 0.011$ & $0.107 \pm 0.006^{\#}$ \\
LA & $0.019 \pm 0.002$ & $0.062 \pm 0.006^{*}$ & $0.033 \pm 0.006$ & $0.035 \pm 0.003^{*}$ \\
HW/BW & $2.72 \pm 0.07$ & $3.82 \pm 0.12^{*}$ & $3.39 \pm 0.14^{*}$ & $4.42 \pm 0.16^{\#}$ \\
\hline
\end{tabular}

Data are presented as mean \pm SEM. CON: healthy control animals; AV: animals with increased pulmonary blood flow due to an aortocaval shunt; MC: animals with increased pulmonary arterial pressure due to monocrotaline injections; MC+AV: animals with both increased pulmonary arterial pressure and increased pulmonary blood flow, receiving both monocrotaline and an aortocaval shunt; RV: right ventricle; IVS: interventricular septum; LV: left ventricle; RA: right atrium; LA: left atrium; HW/BW: heart weight to body weight ratio. *: $p<0.05$ versus control group, $\#$ : $p<0.05$ versus all other groups.

Right atrial weight correlated with echocardiographical diastolic right ventricular internal diameter measured 4 weeks after surgery (Pearson's correlation coefficient $=0.61$; $\mathrm{p}=0.02)$.

Lung weight was significantly increased in both the MC and $\mathrm{MC}+\mathrm{AV}$ group compared with control animals, with no significant additional effect of AV shunt (lung weight: $1.35 \pm 0.06 \mathrm{~g}, 1.72 \pm 0.09 \mathrm{~g}, 2.44 \pm 0.34 \mathrm{~g}$ ( $\mathrm{p}=0.001$ versus $\mathrm{CON}$ ) and $2.36 \pm 0.12 \mathrm{~g}(\mathrm{p}<0.001$ versus $\mathrm{CON})$ in $\mathrm{CON}, \mathrm{AV}, \mathrm{MC}$ and $\mathrm{MC}+\mathrm{AV}$, respectively). 


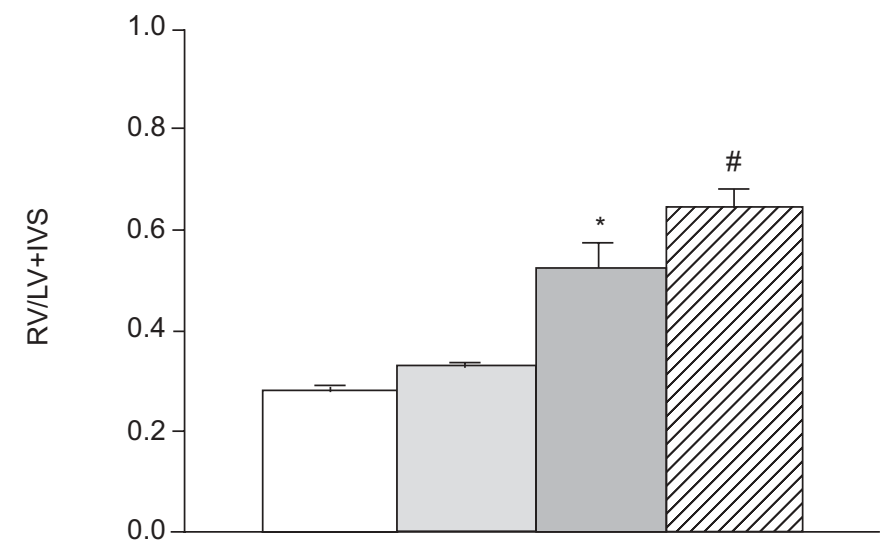

FIGURE 2. Right ventricular (RV)/left ventricular (LV)+interventricular septum (IVS) weight ratio (Futton index) in healthy control animals ( $\square$ ), animals with increased pulmonary blood flow due to an aortocaval shunt ( $\square$ ), animals with increased pulmonary pressure due to monocrotaline injections ( $\square$ ), and animals with increased pulmonary artery pressure and increased pulmonary blood flow, receiving monocrotaline and an aortocaval shunt (घ). *: $p<0.05$ versus controls; * $p<0.05$ versus all other groups.

\section{Histology}

Pre-acinar pulmonary arteries (>50 $\mu \mathrm{m})$

Larger pulmonary arteries increased in both number and dilation in the MC+AV group (table 3). Wall thickness increased both in the $\mathrm{MC}$ and $\mathrm{MC}+\mathrm{AV}$ group. No intimal

\begin{tabular}{|c|c|c|c|c|}
\hline & CON & AV & MC & $\mathrm{MC}+\mathrm{AV}$ \\
\hline \multicolumn{5}{|l|}{$\begin{array}{r}\text { Larger pulmonary } \\
\text { arteries }>50 \mu \mathrm{m}\end{array}$} \\
\hline Outer vessel diameter & $106.8 \pm 5.6$ & $100.7 \pm 9.2$ & $106.1 \pm 6.5$ & $140.0 \pm 4.7^{\star}$ \\
\hline Inner vessel diameter & $88.0 \pm 5.3$ & $75.3 \pm 7.5$ & $78.0 \pm 7.3$ & $110.2 \pm 4.1^{*}$ \\
\hline Medial wall thickness & $9.4 \pm 0.5$ & $12.7 \pm 2.1$ & $14.0 \pm 2.1^{*}$ & $14.9 \pm 0.7^{*}$ \\
\hline Wall:Iumen ratio & $0.12 \pm 0.01$ & $0.20 \pm 0.04$ & $0.24 \pm 0.07^{*}$ & $0.15 \pm 0.01$ \\
\hline Arteries $n$ & $20 \pm 2.0$ & $24 \pm 4.0$ & $38 \pm 6.0$ & $48 \pm 4.0^{*}$ \\
\hline \multicolumn{5}{|l|}{$\begin{array}{l}\text { Smaller pulmonary } \\
\text { vessels }<\mathbf{5 0} \mu \mathrm{m}\end{array}$} \\
\hline Outer diameter & $32.3 \pm 0.7$ & $34.3 \pm 1.5$ & $32.5 \pm 1.0$ & $31.5 \pm 0.6$ \\
\hline Luminal diameter & $31.5 \pm 0.7$ & $32.4 \pm 1.7$ & $26.3 \pm 1.1^{*}$ & $25.7 \pm 0.6^{*}$ \\
\hline Total wall thickness & $0.38 \pm 0.10$ & $0.97 \pm 0.21$ & $3.09 \pm 0.28^{*}$ & $2.89 \pm 0.23^{*}$ \\
\hline Luminal occlusion \% & $3.7 \pm 0.8$ & $8.8 \pm 2.0$ & $30.9 \pm 2.6^{*}$ & $29.8 \pm 2.0^{*}$ \\
\hline \multicolumn{5}{|l|}{ Muscularisation } \\
\hline $\begin{array}{l}\text { Vessel totally } \\
\text { muscularised \% }\end{array}$ & $7.0 \pm 1.9$ & $12.6 \pm 3.6$ & $32.8 \pm 8.4^{\star}$ & $24.0 \pm 2.8^{*}$ \\
\hline $\begin{array}{l}\text { Vessel partially } \\
\text { muscularised \% }\end{array}$ & $0.9 \pm 0.5$ & $0.9 \pm 0.5$ & $3.4 \pm 1.6$ & $5.0 \pm 0.9^{*}$ \\
\hline
\end{tabular}

Data are presented as mean $\mu \mathrm{m} \pm \mathrm{SEM}$. CON: healthy control animals; $\mathrm{AV}$ : animals with increased pulmonary blood flow due to an aortocaval shunt; MC: animals with increased pulmonary arterial pressure due to monocrotaline injections; MC+AV: animals with both increased pulmonary arterial pressure and increased pulmonary blood flow, receiving both monocrotaline and an aortocaval shunt. proliferation could be demonstrated in the pre-acinar arteries of either group.

Intra-acinar pulmonary vessels $(<50 \mu \mathrm{m})$

In contrast to the $\mathrm{CON}$ and $\mathrm{AV}$ group, increased peripheral muscularisation and luminal occlusion due to neo-intimal formation were observed to the same extent in the MC and $\mathrm{MC}+\mathrm{AV}$ groups (table 3; fig. $3 \mathrm{a}$ and $3 \mathrm{~b}$ ). Using immunohistochemistry, this cellular neo-intimal formation stained positive for both eNOS and for $\alpha$-smooth muscle actin (fig. 3c and $3 d)$.

\section{Relating clinical parameters to haemodynamics and vascular histopathology}

Rats with thoracic fluid had: 1) significantly lower body weight (348 \pm 9 versus $398 \pm 9 \mathrm{~g} ; \mathrm{p}=0.001) ; 2)$ higher systolic PAPs $(59 \pm 6$ versus $38 \pm 3 \mathrm{mmHg} ; \mathrm{p}=0.006) ; 3)$ higher cardiac to body weight ratio $\left(4.2 \pm 0.1\right.$ versus $\left.\left.3.2 \pm 0.1 \mathrm{~g} \cdot \mathrm{kg}^{-1} ; \mathrm{p}<0.001\right) ; 4\right)$ higher right ventricular weight ratio $(0.67 \pm 0.03$ versus $0.33 \pm 0.02$; $\mathrm{p}<0.001)$; and 5) more luminal occlusion of the intra-acinar vessels $(31.3 \pm 2.0$ versus $11.4 \pm 2.3 \% ; \mathrm{p}<0.001)$ compared with rats without thoracic fluid.

A significant correlation was observed between luminal occlusion of the intra-acinar pulmonary vessels and right ventricular hypertrophy (expressed as right ventricular/left ventricular+interventricular septum; Pearson's correlation coefficient $=0.78 ; \mathrm{p}<0.001)$.

\section{DISCUSSION}

The aim of the present study was to investigate the role of increased pulmonary blood flow on pulmonary vascular remodelling in pulmonary arterial hypertension. Therefore, an aortacaval shunt was created in monocrotaline-treated rats. The major finding was that compared with nonshunted monocrotaline-treated rats, shunted monocrotaline rats showed increased morbidity and mortality. This was associated with increased pulmonary-to-systemic artery pressure ratios, increased right ventricular hypertrophy and dilatation, and a higher incidence of tricuspid valve regurgitation. Pulmonary vascular histopathology and morphometry revealed no additional effects of flow on the intra-acinar pulmonary vessel remodelling. These findings suggest that the effects of an aortocaval shunt on morbidity and mortality could be attributed to more pronounced right heart failure, rather than to altered intra-acinar pulmonary vessel remodelling.

Pre-acinar pulmonary arteries did increase in number and size compared with both isolated increased pressure as well as isolated increased flow. The present study, however, does not allow differentiation between a secondary dilatatory effect of increased flow and an intrinsic remodelling process of these arteries as a cause for this observation.

\section{Effects of increased pulmonary flow on pulmonary vascular remodelling}

In the current study, rats with isolated increased pulmonary blood flow did not develop changes in PAP and showed a balanced increase in weight of all cardiac compartments. Pulmonary vascular morphometry after 5-6 weeks did not show significant changes compared with control animals. This is congruent with previous reports, where vascular changes 

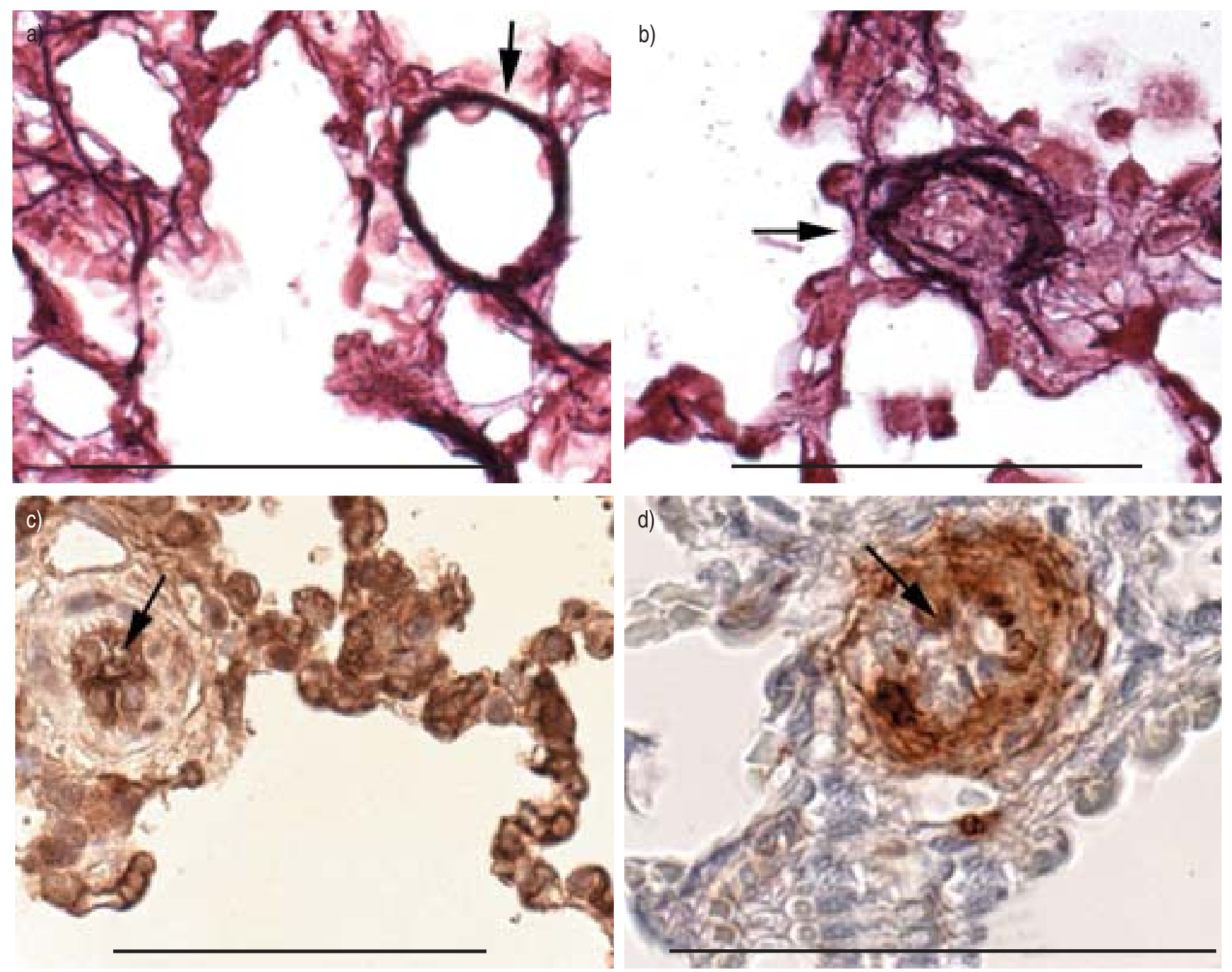

FIGURE 3. Resorcin-fuchsin elastic staining in a) pulmonary intra-acinar vessel in a control animal and b) an animal that both received monocrotaline and an aortocaval shunt. Immunohistochemical staining of neo-intimal formation in the smaller pulmonary vessels of an animal that received both monocrotaline and an aortocaval shunt, c) staining for endothelial nitric oxide synthase and d) $\alpha$-smooth muscle actin. Scale bar $=50 \mu \mathrm{m}$.

usually occur only after much longer periods of exposure to high flow conditions $[8,16]$.

Monocrotaline-treated animals without shunt, displayed increased PAPs and right ventricular hypertrophy, while morphometry revealed increased wall thickness and increased muscularisation in the pre-acinar pulmonary arteries, as expected from earlier studies [3-5]. Interestingly, occlusion of the intra-acinar vessels was also found in monocrotalinetreated rats. These findings are in contrast with previous studies reporting that intima proliferation is caused by the addition of flow in the monocrotaline-treated rats. In these studies, luminal occlusion was not reported in the monocrotaline-treated rats without increased pulmonary flow $[9,11]$. Conversely, the present results are in accordance with NisHIMURA et al. [10], who recently described luminal occlusion in pulmonary arterioles in the monocrotaline rat model. In most of these studies $[10,11,17,18]$ a visual scoring system was used to assess the severity of neo-intimal formation in the smaller pulmonary arteries or the examination was limited to larger pulmonary arteries [9, 19-21], whereas the present study provides qualitative and quantitative morphological characterisation of the pulmonary vasculature.

Animals that received both monocrotaline and a shunt displayed an increased morbidity and mortality compared with all other groups. In this respect, the current results contrast with the conclusion of NISHIMURA et al. [10], who suggested a salvage effect of additional flow in monocrotalinetreated rats using the same model. However, in their experimental design, monocrotaline was injected 1 week after the creation of the aortocaval shunt. Studies in the authors' laboratory showed less pronounced disease development when procedural order was used from the study by NishimURA et al. [10] (data not shown). In the current authors' opinion, this can be explained by the fact that monocrotaline is metabolised in the liver into toxic, but unstable, metabolites [22]. These toxic metabolites have a half-life of a few seconds or 
less. Therefore, they have to exert their toxic effect in the initial passage through the lungs. Increased flow by an aortocaval shunt will lower the concentration of the metabolites transported to the lung. This may explain why induction of a shunt before the administration of monocrotaline will lead to a diminished effect of monocrotaline and less advanced pulmonary vascular disease.

The nature and severity of the histological changes in the present study are comparable with those reported in other combination models of high pulmonary pressure and flow in the rat $[6,9,11]$. However, the origin of the luminal occlusion of intra-acinar vessels, which is often referred to as neo-intima, is less clear. In the present study, the intraluminal occlusion consisted of cellular material. Both endothelial cells and vascular smooth muscle cells were present in this neo-intima, since it consisted of cells that stained positive for eNOS and of cells that stained positive for $\alpha$-smooth muscle actin.

\section{Effects of increased pulmonary flow on the heart}

Although PAP in shunted monocrotaline rats was comparable with values in nonshunted monocrotaline rats, systemic blood pressure was lower. The lower systemic blood pressures could be attributed to a general decrease in cardiac output at the time of sacrifice. The pronounced right ventricular hypertrophy and increased right to left atrial weight ratios do suggest that right ventricular failure, rather than biventricular high output failure, was the major determinant of heart failure.

Echocardiography in rats was established as a useful diagnostic tool for the cardiac assessment of abnormal pulmonary haemodynamics, studying right ventricular wall thickness and ventricular dimensions. The echocardiographical findings of progressive right ventricular dilatation and the development of tricuspid regurgitation in the shunted monocrotaline-treated animals support the diagnosis of right ventricular failure in these animals.

Progression of clinical deterioration and right ventricular hypertrophy were correlated with increased luminal occlusion of the small pulmonary vessels, indicating that the development of right ventricular failure is aggravated by the detrimental combination of increased volume load and increased afterload of the right ventricle.

Taken together, the results suggest that increased morbidity and mortality in the shunted monocrotaline rats could be attributed to effects on the heart, rather than to altered pulmonary vascular remodelling.

\section{Limitations of the study}

In the present model, monocrotaline could be regarded as a confounding factor to flow, because its metabolites induce direct pulmonary endothelial cell damage that might possibly obscure the effects of flow on these endothelial cells.

However, neo-intimal formation, a key feature in pulmonary vascular remodelling in pulmonary arterial hypertension, is induced in this model. Other models, creating left to right shunts in young animals [16, 23, 24] might have advantages with respect to affecting flow and pressure in the pulmonary arteries comparable with the situation in congenital heart disease. However, the increase in PAP in these models is moderate and, until now, they failed to display the more advanced lesions of pulmonary arterial hypertension. These lesions distinguish pulmonary arterial hypertension from other forms of pulmonary hypertension and, therefore, probably form an important clue in the pathophysiology of pulmonary arterial hypertension.

Furthermore, the present study does not provide an answer to the question as to whether increased pulmonary flow induced specific vascular cell responses at a molecular level, which might explain why in congenital heart defects the advanced pulmonary vascular lesions of pulmonary arterial hypertension occur almost exclusively in the presence of increased pulmonary blood flow.

\section{Conclusion}

The present study aimed to investigate the role of increased flow in flow-associated pulmonary hypertension. Detrimental effects of increased pulmonary blood flow in the aortacavalshunted monocrotaline rat model were demonstrated by increased morbidity and mortality. These effects could, however, not be attributed to histopathological aggravation of pulmonary vascular remodelling. In contrast to earlier reports, the neo-intimal reaction described in the shunted monocrotaline-treated rats in the present study was not identified as a specific effect of the increased pulmonary flow, as it was demonstrated to also be present in rats treated with monocrotaline solely.

In conclusion, haemodynamical, echocardiographical and pathological data suggested pronounced right ventricular failure to be the cause for the increased morbidity and mortality, rather than altered pulmonary vascular remodelling. Further research on the specific role of increased pulmonary blood flow in the development of advanced pulmonary vascular lesions in pulmonary arterial hypertension, associated with congenital heart defects, is needed to understand the pathogenesis of this progressive pulmonary vascular disease.

\section{REFERENCES}

1 Geiger R, Berger RM, Hess J, Bogers AJ, Sharma HS, Mooi WJ. Enhanced expression of vascular endothelial growth factor in pulmonary plexogenic arteriopathy due to congenital heart disease. J Pathol 2000; 191: 202-207.

2 Wagenvoort CA, Mooi WJ. Plexogenic arteriopathy. In: Munro Neville A, Walker F, Gottlieb LS, eds. Biopsy pathology of the pulmonary vasculature. London, Chapman and Hall Medical, 1989; pp 56-113.

3 van Suylen RJ, Smits JF, Daemen MJ. Pulmonary artery remodeling differs in hypoxia- and monocrotaline-induced pulmonary hypertension. Am J Respir Crit Care Med 1998; 157: 1423-1428.

4 Todorovich-Hunter L, Dodo H, Ye C, McCready L, Keeley FW, Rabinovitch M. Increased pulmonary artery elastolytic activity in adult rats with monocrotalineinduced progressive hypertensive pulmonary vascular disease compared with infant rats with nonprogressive disease. Am Rev Respir Dis 1992; 146: 213-223.

5 Guzowski DE, Salgado ED. Changes in main pulmonary artery of rats with monocrotaline-induced pulmonary hypertension. Arch Pathol Lab Med 1987; 111: 741-745. 
6 Botney MD. Role of hemodynamics in pulmonary vascular remodeling: implications for primary pulmonary hypertension. Am J Respir Crit Care Med 1999; 159: 361-364.

7 Qi J, Du J, Tang X, Li J, Wei B, Tang C. The upregulation of endothelial nitric oxide synthase and urotensin-II is associated with pulmonary hypertension and vascular diseases in rats produced by aortocaval shunting. Heart Vessels 2004; 19: 81-88.

8 Dai ZK, Tan MS, Chai CY, Chen IJ, Jeng AY, Wu JR. Effects of increased pulmonary flow on the expression of endothelial nitric oxide synthase and endothelin-1 in the rat. Clin Sci (Lond) 2002; 103: Suppl. 48, 289S-293S.

9 Tanaka Y, Schuster DP, Davis EC, Patterson GA, Botney MD. The role of vascular injury and hemodynamics in rat pulmonary artery remodeling. J Clin Invest 1996; 98: 434-442.

10 Nishimura T, Faul JL, Berry GJ, Kao PN, Pearl RG. Effect of a surgical aortocaval fistula on monocrotaline-induced pulmonary hypertension. Crit Care Med 2003; 31: 1213-1218.

11 Okada K, Tanaka Y, Bernstein M, Zhang W, Patterson GA, Botney MD. Pulmonary hemodynamics modify the rat pulmonary artery response to injury. A neointimal model of pulmonary hypertension. Am J Pathol 1997; 151: 1019-1025.

12 Garcia R, Diebold S. Simple, rapid, and effective method of producing aortocaval shunts in the rat. Cardiovasc Res 1990; 24: 430-432.

13 Kato Y, Iwase M, Kanazawa H, et al. Progressive development of pulmonary hypertension leading to right ventricular hypertrophy assessed by echocardiography in rats. Exp Anim 2003; 52: 285-294.

14 Rabinovitch M, Gamble W, Nadas AS, Miettinen OS, Reid L. Rat pulmonary circulation after chronic hypoxia: hemodynamic and structural features. Am J Physiol 1979; 236: H818-H827.
15 Proctor GB, Horobin RW. Chemical structures and staining mechanisms of Weigert's resorcin-fuchsin and related elastic fiber stains. Stain Technol 1988; 63: 101-111.

16 Rondelet B, Kerbaul F, Motte S, et al. Bosentan for the prevention of overcirculation-induced experimental pulmonary arterial hypertension. Circulation 2003; 107: 1329-1335.

17 Nishimura T, Faul JL, Berry GJ, et al. Simvastatin attenuates smooth muscle neointimal proliferation and pulmonary hypertension in rats. Am J Respir Crit Care Med 2002; 166: 1403-1408.

18 Faul JL, Nishimura T, Berry GJ, Benson GV, Pearl RG, Kao PN. Triptolide attenuates pulmonary arterial hypertension and neointimal formation in rats. Am J Respir Crit Care Med 2000; 162: 2252-2258.

19 Colice GL, Hill N, Lee YJ, et al. Exaggerated pulmonary hypertension with monocrotaline in rats susceptible to chronic mountain sickness. J Appl Physiol 1997; 83: 25-31.

20 Stenmark KR, Morganroth ML, Remigio LK, et al. Alveolar inflammation and arachidonate metabolism in monocrotaline-induced pulmonary hypertension. Am J Physiol 1985; 248: H859-H866.

21 Todd L, Mullen M, Olley PM, Rabinovitch M. Pulmonary toxicity of monocrotaline differs at critical periods of lung development. Pediatr Res 1985; 19: 731-737.

22 Huxtable RJ. Activation and pulmonary toxicity of pyrrolizidine alkaloids. Pharmacol Ther 1990; 47: 371-389.

23 Black SM, Fineman JR, Steinhorn RH, Bristow J, Soifer SJ. Increased endothelial NOS in lambs with increased pulmonary blood flow and pulmonary hypertension. Am J Physiol 1998; 275: H1643-H1651.

24 Jouannic JM, Roussin R, Hislop AA, et al. Systemic arteriovenous fistula leads to pulmonary artery remodeling and abnormal vasoreactivity in the fetal lamb. Am J Physiol Lung Cell Mol Physiol 2003; 285: L701-L709. 